\title{
Uma maquete da estrutura em treliças simples triangulares para o ensino de estática
}

\author{
A simple triangular trusses structure model for statics teaching
}

\author{
Rodrigo Braga de Farias ${ }^{\dagger 1}$, V. L. B. de Jesus ${ }^{1}$, A. L. de Oliveira ${ }^{* 10}$ \\ ${ }^{1}$ Instituto Federal de Educação, Ciência e Tecnologia do Rio de Janeiro, Campus Nilópolis, Nilópolis, RJ, Brasil.
}

Recebido em 10 de Abril de 2020. Revisado em 25 de Junho de 2020. Aceito em 07 de Julho de 2020.

\begin{abstract}
Este trabalho apresenta um recurso didático experimental em alternativa aos clássicos experimentos para verificação da lei de Hooke e daqueles que utilizam sensores eletrônicos para o estudo de forças atuantes em estruturas estáticas. Desenvolvemos uma maquete de uma estrutura típica em treliças triangulares simples para pontes, com molas acopladas em cada barra da estrutura. Com essa maquete, é possível explorar as potencialidades para a obtenção de resultados qualitativos e quantitativos sobre as forças que atuam nas barras da estrutura. As molas acopladas às barras da estrutura tem o propósito de nos permitir a visualização do comportamento de compressão ou tração e obter dados por medição direta das forças axiais atuantes em cada barra utilizando a lei de Hooke. Comparamos os valores experimentais das forças axiais de cada barra por medição direta com os resultados obtidos pelo método dos nós. Os resultados qualitativos e quantitativos dessa comparação indicam a viabilidade da utilização da maquete para fins didáticos.
\end{abstract}

Palavras-chave: Instrumentação para o ensino, Desenvolvimento de material didático, Estática, Estrutura em treliças, Força elástica.

This work presents an experimental teaching resource as an alternative to the classic experiments to verify Hooke's law and those that use electronic sensors to study forces acting on static structures. We developed a model of a typical structure in simple triangular trusses for bridges, with springs attached to each member of the structure. With this model, it is possible to explore the potential for obtaining qualitative and quantitative results on the forces acting on the members of the structure. The springs coupled to the structure's bars have the purpose of allowing us to visualize the compression or traction behavior and obtain data by direct measurement of the axial forces acting on each member using Hooke's law. We compare the experimental values of the axial forces of each member by direct measurement with the results obtained by the method of joints. The qualitative and quantitative results of this comparison indicate the feasibility of using the model for educational purposes.

Keywords: Instrumentation for teaching, Development of teaching material, Statics, Truss structures, Elastic force.

\section{Introdução}

Apresentar a ciência como um empreendimento humano, melhorar sua compreensão e desempenho da sociedade para os estudantes, é um dos objetivos da abordagem em Ciência, Tecnologia e Sociedade (CTS) para o ensino. A análise interdisciplinar da ciência e da tecnologia no contexto social é um dos pilares do enfoque CTS [1].

A discussão sobre o enfoque CTS e alfabetização científica e tecnológica $(\mathrm{ACT})$ iniciou há quase meio século e continua até os dias de hoje, mas a prática dessas teorias pedagógicas não atingiram sua plenitude [2]. A experimentação como ferramenta pedagógica tem diferentes abordagens que possam completar as expectativas e objetivos [3], portanto, a experimentação investigativa e problematizadora pode ser uma estratégia para criar

\footnotetext{
${ }^{\dagger}$ Discente da Licenciatura em Física do IFRJ, Campus Nilópolis.

*Endereço de correspondência: alexandre.oliveira@ifrj.edu.br.
}

condições para professores integrarem o enfoque CTS em suas práticas.

O ensino sob a perspectiva CTS apoiado na experimentação como estratégia didática, pode contribuir para a formação de estudantes críticos, capazes de refletir, questionar e debater sobre a ciência, a tecnologia e sua relação com a sociedade [4]. Deve-se destacar que o experimento apresentado no presente artigo poderá oportunizar a ampliação do significado da experimentação para além do laboratório didático, promovendo a compreensão de uma estrutura em treliças que possui significado nas vidas cotidianas dos alunos, a saber: uma ponte. Numa perspectiva CTS, essa experimentação pode ser explorada sob a relação entre Ciência, Engenharia e Sociedade, por meio de discussões sobre Física, atreladas com questões sociais como, por exemplo, impactos das pontes em zonas urbanas. É importante considerar que oportunidades de "mão na massa" podem ser convertidas em experiências de aprendizagem como apontado na Ref. [5]. 
Como destacado na Ref. [6] e citações no seu texto, as estruturas em treliças são utilizadas em várias tecnologias tradicionais (pontes, postes elétricos, guindastes, veículos), em aplicações inovadoras (nanotreliças), para o entendimento de algumas estruturas biológicas (esqueletos de vertebrados, materiais proteicos) e modelos conceituais (redes cristalinas).

Para facilitar o entendimento da mecânica de uma estrutura em treliças, na Ref. [6] é apresentada uma maquete didática em estrutura em aço inox com sistemas de amortecimento conectados às estruturas usinadas em tubos de alumínio e molas helicoidais construídas com cordas musicais. No modelo apresentado na Ref. [6] é possível visualizar o comportamento global da estrutura bem como medições diretas e altamente precisas da tração/compressão da barra por conta de um "pointer" acoplado ao sistema para algumas barras da estrutura e as comparando com resultados teóricos. É preciso enfatizar que tal maquete foi desenvolvida em um grupo de pesquisa do departamento de engenharia mecânica e estrutural de uma universidade italiana, cujo objetivo principal é se dedicar a projetos altamente elaborados de pesquisa nessa área. Em outras palavras, o acesso a equipamentos de usinagem e material é algo corriqueiro no contexto dos autores desse artigo.

Este trabalho tem como objetivo explorar as potencialidades de uma maquete didática de estrutura treliçada construída com materiais de fácil acesso para o estudo de estática, evitando tanto montagens sofisticadas quanto a utilização de usinagem mecânica. Especificamente, pretendemos verificar qualitativamente a ação das forças na estrutura inserindo pequenas molas nas barras em diferentes posições da treliça, e ainda verificar quantitativamente, por medida direta da distensão ou compressão de cada mola obtendo o valor de cada força via lei de Hooke. As medidas obtidas por medição direta são comparadas com os resultados obtidos pelo método dos nós.

Apresentamos a seguir o escopo deste trabalho. $\mathrm{Na}$ seção 2, apresentamos elementos e aspectos sobre uma estrutura em treliças. Na seção 3, descrevemos a metodologia aplicada para a confecção da nossa maquete experimental de uma ponte treliça simples triangular. Na seção 4, comparamos os resultados dos esforços em cada barra da estrutura pelo método dos nós e por medição direta por meio da lei de Hooke a fim de testar a viabilidade da utilização da maquete didática experimental para resultados qualitativos e quantitativos. Por fim, na seção 5, apresentamos as conclusões.

\section{Estruturas em Treliças}

O nome treliça deve-se ao fato de todos os elementos do conjunto pertencerem a um único plano, chamado também de treliças planas.

As treliças são estruturas cujo elemento de construção são barras ligadas entre si, sob forma geométrica triangular e que visam formar uma estrutura rígida que resista a esforços, somente normais. Elas formam um tipo de estrutura que é muito eficaz a esforços e tem diversas aplicações. Pontes, viadutos, guindastes, coberturas são algumas de suas aplicações.

Uma treliça consiste de barras retas ligadas em juntas (nós). Essas barras são interligadas apenas em suas extremidades, assim nenhuma barra é contínua através de uma junta (nó) [7].

Na Figura 1 estão apresentadas as formas e classificações das treliças simples triangulares utilizadas em construções de pontes.

Uma treliça triangular simples como aquelas ilustradas na Figura 1, é formada a partir de uma estrutura triangular básica, constituída de 3 barras e 3 nós. Para cada duas novas barras acrescentadas, o número de nós aumenta de uma unidade. Portanto, é possível deduzir que o número de barras $b$ é dado por

$$
b=2 n 3,
$$

em que $n$ é o número total de nós.

Na Figura 2 está destacada a ilustração da estrutura em treliça Warren para ponte, a do mesmo tipo utilizado no ensaio deste trabalho com as indicações e denominações de cada elemento da estrutura.

A estrutura ilustrada na Figura 2 é de treliça simples bidimensional e bi-apoiada. Numa ponte, por exemplo, a estrutura é composta de duas treliças e são unidas por barras transversais. Essas barras transversais são

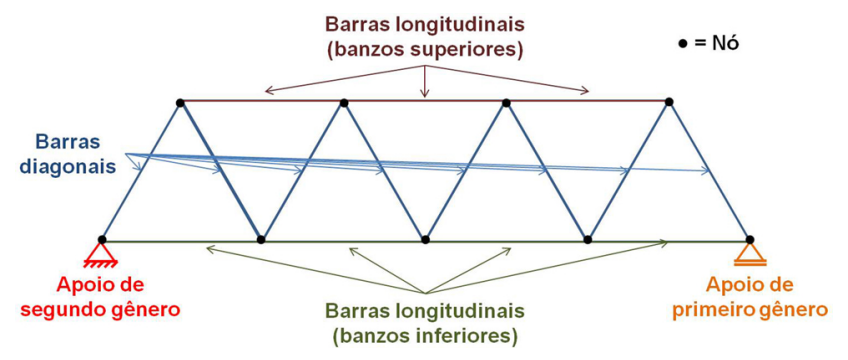

Figura 2: Treliça do tipo Warren com indicação e nomenclatura de seus elementos estruturais.

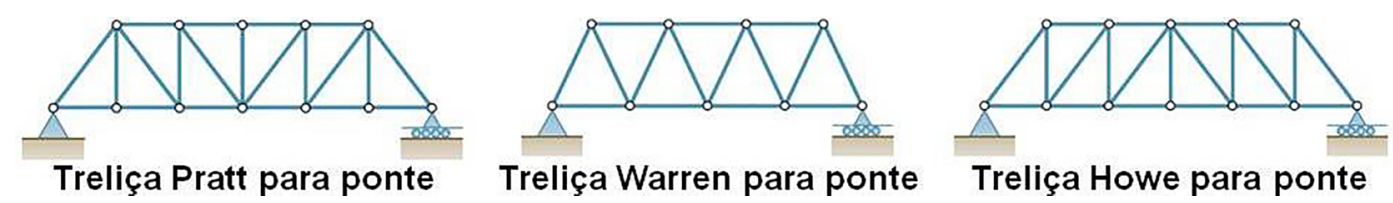

Figura 1: Classificações dos tipos mais comuns de treliças simples triangulares utilizadas em construções de pontes. 
chamadas de contraventos (ver Figura 3), as quais têm a função de impedir a rotação da estrutura (torção).

$\mathrm{Na}$ Figura 3, chamamos cada estrutura em treliça de vigas. Portanto, temos a viga 1 e a viga 2 unidas pelos contraventos.

Conhecidos os nomes dos elementos de uma estrutura em treliça, a seção 3 está dedicada ao cálculo das forças que atuam sobre as barras diagonais e longitudinais devido a uma carga adicional $\vec{P}$ posicionada no ponto médio do contravento central.

\section{Cálculo das Forças Sobre as Barras}

As treliças podem ser consideradas como uma estrutura agrupada em nós e barras. Essas barras estão submetidas a duas forças, uma em cada extremidade, onde se encontram os nós. A terceira lei de Newton indica que a força de ação de uma barra que atua sobre o nó tem a mesma intensidade da força de reação que atua sobre o nó sobre a barra, a mesma direção porém em sentidos opostos.

As forças que atuam sobre a barra $\left(\vec{F}_{b}\right)$ podem ter o efeito de compressão ou tração que podem atuar ao longo de uma barra de treliça. Quando essas forças tendem a encurtar a barra, isto é, tendem a comprimir a barra, é por convenção admitida como negativa. Por outro lado, quando essas forças tendem a esticar a barra, isto é, tendem a tracionar a barra, é por convenção admitida como positiva. A Tab. 1 resume essa convenção.

Para verificarmos a estabilidade da estrutura e calcular as forças sobre as barras após a introdução de uma carga adicional $(\vec{P})$ à estrutura, utilizaremos três passos, a saber: verificar a condição isostática da treliça; calcular as reações de apoio $\vec{R}$ e calcular as forças exercidas sobre as barras pelo método dos nós.

A condição isostática da treliça fica estabelecida quando as reações são estaticamente determinadas e a treliça rí-

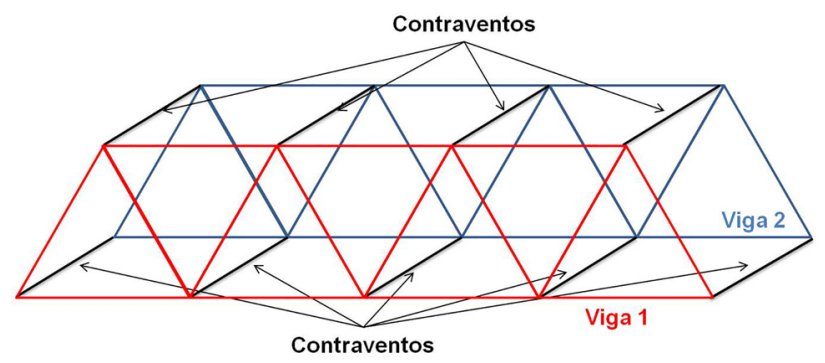

Figura 3: Representação de duas estruturas treliçadas (vigas) unidas por contraventos.

Tabela 1: Convenção adotada para os efeitos da força de ação que atua sobre a barra.

\begin{tabular}{lcc}
\hline Efeito & Esquema & Força na barra \\
\hline Tração & barra & $+\left|\vec{F}_{b}\right|$ \\
Compressão & barra & $-\left|\vec{F}_{b}\right|$ \\
\hline
\end{tabular}

gida ${ }^{1}$ é impossibilitada de mover-se por translação ou rotação.

Em engenharia, o apoio de primeiro gênero (indicado na Figura 2, à direita) como trata-se de um "apoio livre" a força de apoio na horizontal é zero e portanto, nesse ponto, só há a representação da força de apoio na vertical $\left(\vec{R}_{v B}\right)$. Para o apoio de segundo gênero (indicado na Figura 2, à esquerda), como trata-se de um "apoio fixo", há a representação tanto a força de apoio na vertical $\left(\vec{R}_{v A}\right)$ quanto a força de apoio na horizontal $\left(\vec{R}_{h A}\right)$.

No entanto, dos conceitos gerais sobre o equilíbrio dos corpos, para que a estrutura permaneça estável é necessário que se satisfaça tanto o equilíbrio de translação quanto o de rotação. O equilíbrio de translação é satisfeito quando o somatório de forças que atuam sobre o corpo é nula, isto é,

$$
\sum \vec{F}=0,
$$

e o equilíbrio de rotação quando o somatório dos momentos das forças que atuam no corpo também for nula, isto é,

$$
\sum \vec{M}_{F}=0
$$

Portanto, uma vez que a treliça em questão é isostática, na direção horizontal, temos que a força de reação de apoio na horizontal no ponto $\mathrm{A}\left(\vec{R}_{h A}\right)$ é nula, satisfazendo a Equação (2) para a essa direção, em módulo,

$$
R_{h A}=0
$$

Na direção vertical, foi introduzida uma carga adicional $(\vec{P})$ no ponto médio do contravento central inferior (ver Figura 3). Ao satisfazer as condições de equilíbrio, Equação (2) e Equação (3), temos em módulo que a força de reação de apoio na vertical no ponto $\mathrm{A}\left(\vec{R}_{v A}\right)$ e a força de reação de apoio na vertical no ponto $\mathrm{B}\left(\vec{R}_{v B}\right)$ são iguais,

$$
\vec{R}_{v A}=\vec{R}_{v B}=\vec{R}_{v}
$$

tal que, em módulo,

$$
R_{v}=\frac{P}{2}
$$

Uma das formas para se determinar as forças em cada barra é a utilização do método dos nós. A análise de uma treliça se reduz ao cálculo das forças em suas várias barras e à determinação do efeito em cada barra, ou seja, se cada barra está sujeita à tração ou compressão.

Na Figura 4, apresentamos a estrutura Warren com os nós e as barras numeradas, a carga adicional $\vec{P}$ indicada no nó 3, bem como as forças de reações de apoio.

As forças que atuam nas barras foram obtidas pelo método dos nós e estão detalhadas no Apêndice A. A Tabela 2 reúne os resultados dessas análises, em que a numeração das barras (b) segue àquela indicada na Figura 4.

\footnotetext{
1 O termo "rígida" é para indicar que a treliça não entrará em colapso.
} 
Tabela 2: Intensidade das forças $\left(F_{b}\right)$ que atuam em cada barra $b(b=1$ a 15$)$ indicando o comportamento de compressão ou tração na situação ilustrada na Figura 4.

\begin{tabular}{lccc}
\hline & Barra $(b)$ & Força nas barras $\left(F_{b}\right)$ & Comportamento \\
\hline Barras longitudinais & 11 & $F_{11}=-P$ & Compressão \\
superiores (Banzos & 13 & $F_{13}=-2 P$ & Compressão \\
superiores) & 15 & $F_{15}=-P$ & Compressão \\
\hline Barras diagonais & 2 & $F_{2}=\frac{-\sqrt{2}}{2} P$ & Compressão \\
& 9 & $F_{9}=\frac{+\sqrt{2}}{2} P$ & Tração \\
& 4 & $F_{4}=\frac{-\sqrt{2}}{2} P$ & Compressão \\
& 10 & $F_{10}=\frac{+\sqrt{2}}{2} P$ & Tração \\
& 6 & $F_{6}=\frac{+\sqrt{2}}{2} P$ & Tração \\
& 12 & $F_{12}=\frac{-\sqrt{2}}{2} P$ & Compressão \\
& 8 & $F_{8}=\frac{ \pm \sqrt{2}}{2} P$ & Tração \\
& 14 & $F_{14}=\frac{-\sqrt{2}}{2} P$ & Compressão \\
\hline Barras Longitudinais & 1 & $F_{1}=\frac{+1}{2} P$ & Tração \\
inferiores (Banzos & 3 & $F_{3}=\frac{+3}{2} P$ & Tração \\
inferiores) & 5 & $F_{5}=\frac{+3}{2} P$ & Tração \\
& 7 & $F_{7}=\frac{+1}{2} P$ & Tração \\
\hline
\end{tabular}

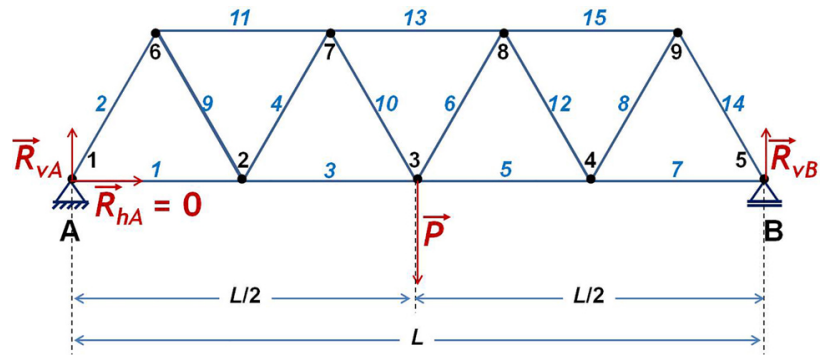

Figura 4: Representação de uma estrutura em treliça Warren de comprimento $L$ apoiada nos pontos A e B, com carga adicional $\vec{P}$ no ponto médio na reta que liga os pontos $A$ e $B$. Em algarismos pretos estão numerado os nós, em algarismos azuis e em itálico a numeração das barras, em vermelho as reações devido ao apoio $\left(\vec{R}_{v A} \quad=\right.$ reação vertical no ponto $\mathrm{A}, \vec{R}_{v B}$ = reação vertical no ponto B) e a carga adicional $\vec{P}$. Como o ponto $A$ trata-se de um apoio de segundo gênero a reação horizontal no ponto $A$ nula $\left(\vec{R}_{h A}=0\right)$ foi representada.

\section{Montagem da Maquete Didática}

A elaboração deste experimento utilizando uma maquete didática tem a finalidade de facilitar a visualização sobre o comportamento das forças em uma estrutura treliçada e poder obter os valores dessas forças por medida direta utilizando a lei de Hooke. A representação esquemática da maquete está ilustrada na Figura 5.

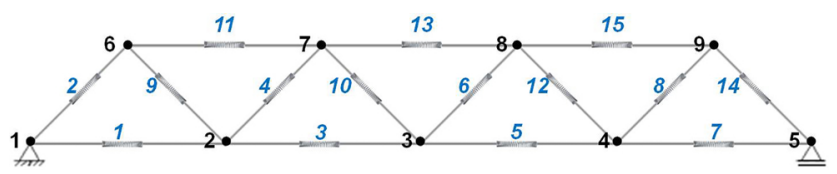

Figura 5: Representação esquemática da maquete didática. Em algarismos pretos estão numerados os nós e em azul, junto às molas inseridas na estrutura, representam a numeração das barras.
Na Tabela 3 estão apresentados os materiais utilizados para construção da maquete didática de treliças, quantidades e os valores dos custos.

São necessários ainda um instrumento para medição (régua, metro, trena ou paquímetro), para o corte dos palitos (alicate de corte, tesoura), lápis para marcação dos nós e dois pedaços de tubo de PVC de $25 \mathrm{~mm}$ com $15 \mathrm{~cm}$ de comprimento.

Na presente maquete didática inserimos uma mola em cada barra da treliça, para as duas estruturas, num total de 30 molas. As molas foram extraídas de canetas esferográficas paper mate da marca Kilométrica 100 RT 1.0 M. Dessa forma, é possível obter medidas de tração/compressão razoavelmente confiáveis, evitando um gasto com material e usinagem para confeccionar um "pointer", como foi apresentado na Ref. [6].

A constante elástica da mola $(k)$ foi obtida escolhendose três molas ao acaso e, pela lei de Hooke, encontramos o valor $k=325,8(1) \mathrm{N} / \mathrm{m}$.

Uma vez conhecida a constante elástica da mola, foi montada uma estrutura treliçada com varetas de bambu, aquelas utilizadas para confecção de pipas (papagaio, cafifas), no intuito de demonstrar que um material tão flexível pode se tornar bastante resistente em uma estrutura de treliça. As varetas de bambu foram adquiridas com dimensões de $45 \mathrm{~cm}$ de comprimento e diâmetro médio de $3 \mathrm{~mm}$. Na montagem, adotamos nas barras

Tabela 3: Material utilizado e custo referente a maio de 2019.

\begin{tabular}{lll}
\hline Quantidade & Material & Valor total \\
\hline 30 & Varetas de bambu & $\mathrm{R} \$ 3,00$ \\
1 & Pistola para cola quente & $\mathrm{R} \$ 7,50$ \\
5 & Baguetes de cola & $\mathrm{R} \$ 3,75$ \\
1 & Régua & $\mathrm{R} \$ 1,50$ \\
1 & Tubo de PVC $25 \mathrm{~mm}$ & $\mathrm{R} \$ 13,90$ \\
1 & Lápis & $\mathrm{R} \$ 1,00$ \\
30 & Molas & $\mathrm{R} \$ 51,00$ \\
Total & & $\mathrm{R} \$ 75,65$ \\
\hline
\end{tabular}


superiores comprimento de 60,00(5) cm, nas barras inferiores $92,00(5) \mathrm{cm}$ e nas diagonais verticais $13,50(5) \mathrm{cm}$. Utilizamos uma pistola de cola quente no processo de montagem para unir as duas vigas treliçadas em forma de ponte, unimos as duas barras inferiores e as duas barras superiores com barras transversais horizontais nas mesmas dimensões das diagonais verticais chamados na engenharia de contraventos com 10,00(5) cm de comprimento. Os contraventos são responsáveis para minimizar os esforços de torção, provocados pela carga nela submetidas ou por ventos. Em nosso caso, somente pela carga.

As barras inferiores estão apoiadas em suas extremidades em dois tubos de PVC de $25 \mathrm{~mm}$ de diâmetro com 15,00(5) cm de comprimento, exemplificando pilares reais de uma ponte, ficando um vão-livre de $80,00(5) \mathrm{cm}$ entre apoios. As barras diagonais verticais e horizontais formaram um ângulo de $45,0(5)^{\circ}$ entre as diagonais e as barras inferiores e superiores em todos os triângulos, podendo ser classificados por treliças do tipo Warren.

Em cada barra vertical e horizontal foi colocada uma mola, substituindo uma seção da barra por uma seção de mesmo comprimento da mola.

Apresentamos na Figura 6 a maquete didática de treliças Warren descrita confeccionada.

Para exemplificar uma treliça metálica real, tivemos o cuidado de montar a estrutura em bambu com o máximo de rigor possível nas marcações para ser o mais fiel às medições. Nesse caso, não observaremos a resistência da estrutura até sua ruptura, ou seja, o quanto de carga adicional suporta, e sim, a observação do comportamento de tração ou compressão nas barras bem como a obtenção das forças exercidas sobre cada barra. A vantagem da utilização desta maquete em relação a uma maquete mais sofisticada como aquela apresentada na Ref. [6], é o fácil acesso ao material e a possibilidade de montar a maquete tornando dispensável qualquer serviço de usinagem em aço inox.

\section{Resultados e Discussões das Forças Exercidas Sobre Barras}

Trataremos nesta seção os resultados obtidos por duas maneiras. A primeira por meio de cálculos utilizando o

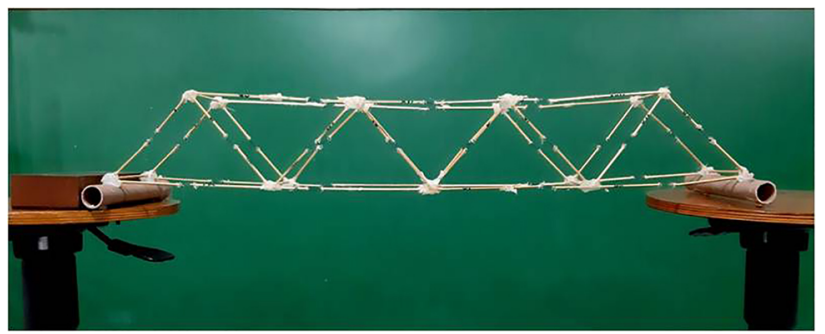

Figura 6: Maquete experimental de estrutura treliçada sem carga adicional. método dos nós e a segunda os resultados experimentais obtidos por medição direta das distensões ou contrações das molas na maquete didática e, posteriormente, os valores experimentais das forças atuantes utilizando a lei de Hooke. Compararemos os resultados com a finalidade de testar a viabilidade da maquete didática para observação do comportamento das forças de compressão e tração na estrutura, bem como medir a intensidade dessas forças em cada barra.

Para a carga adicional, uma massa de 0,05345(1) kg foi posicionada no ponto médio do contravento central inferior. Adotando o valor para a gravidade ${ }^{2}$ de $9,7877394(2)$ $\mathrm{m} / \mathrm{s}^{2}$, temos para a carga adicional na estrutura $P_{\text {total }}=$ 0,5233(1) N. Essa carga adicional é distribuída para as duas vigas, sendo assim, temos a metade do valor para cada viga, isto é, temos uma carga adicional $P=$ $0,2616(1) \mathrm{N}$ por viga.

Estabelecendo as condições da seção 3, utilizamos o método dos nós para determinação das forças normais nas barras diagonais e longitudinais. Lembrando que este método é utilizado para determinar os valores das forças em estruturas rígidas, tendo sempre duas incógnitas em cada nó para resolução do problema.

Fazendo uso das análises indicadas na Tab. 2, obtivemos os resultados conforme as Tabela 4, para a viga 1 e a viga 2 . Nesse caso das vigas, somente para demonstrar a equivalência dos resultados devido à simetria da estrutura. Na Tabela 4, estão apresentados os resultados dos cálculos das forças sobre as barras diagonais e longitudinais, bem como o efeito de compressão $(-)$ ou tração $(+)$.

A intensidades das forças sobre as barras $F_{b}$ por medição direta estão relacionas à lei de Hooke. Portanto, precisa-se conhecer os valores dos comprimentos das molas sem carga adicional $x_{i}$ (ver a Figura 6) e dos comprimento das molas após a introdução da carga adicional $x_{f}$ (ver a Figura 7 ).

Na situação ilustrada na Figura 7, as molas sofrem as ações das forças axiais, as molas se comprimem ou se tracionam e mede-se então, o novo comprimento de deformação $x_{f}$.

As medições de $x_{i}$ e $x_{f}$ foram tomadas utilizando um paquímetro para cada uma das 30 molas nas barras

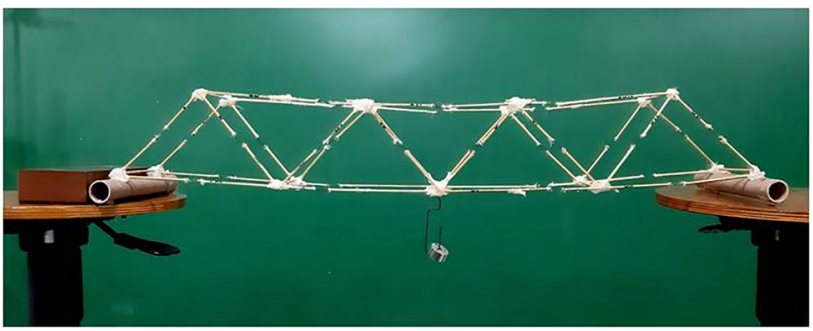

Figura 7: Maquete experimental com carga adicional no ponto médio do contravento central.

\footnotetext{
${ }_{2}$ Valor da aceleração da gravidade local medida pelo observatório nacional.
} 
Tabela 4: Resultados das forças sobre as barras obtidos por meio do método dos nós. As expressões para $F_{b}$ estão na Tabela 2 para uma carga adicional $P=0,2616(1) \mathrm{N}$, aplicada na posição do contravento central inferior. A incerteza $\delta F_{b}= \pm 0,0001 \mathrm{~N}$.

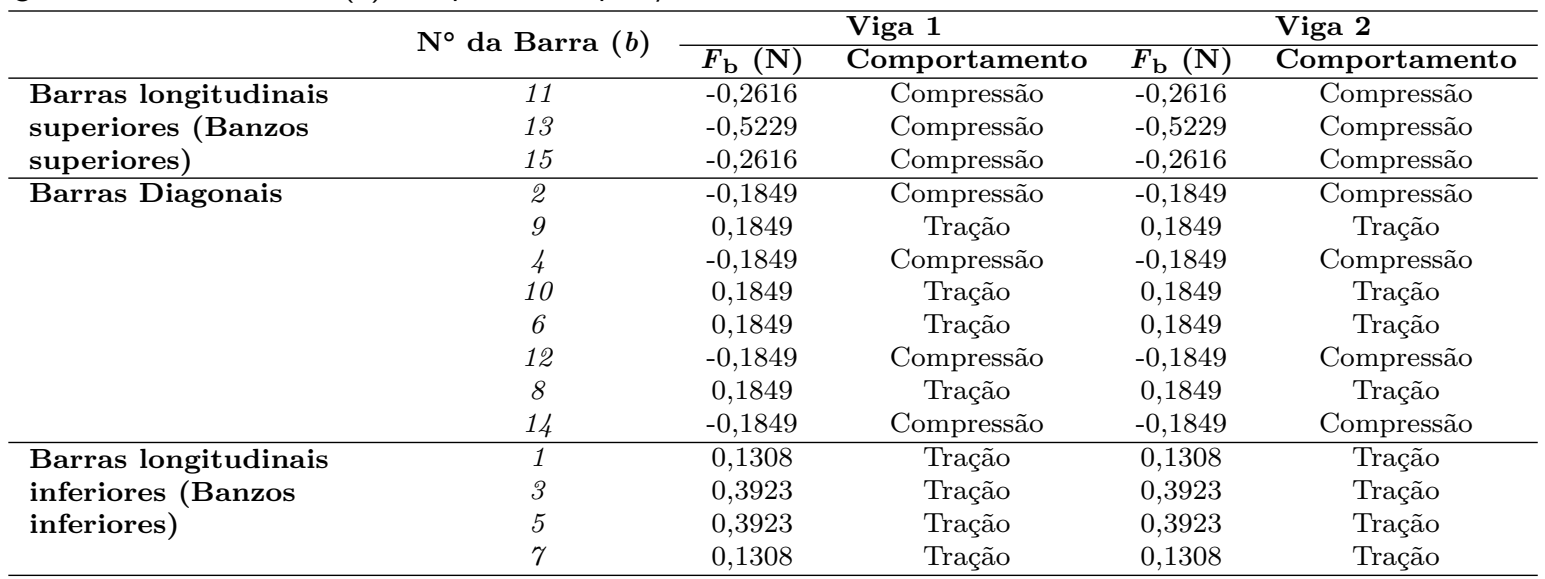

diagonais e nas barras longitudinais nas duas estruturas em treliças (viga 1 e viga 2). A Figura 8 apresentam as medições de $x_{i}$ (Figura 8(a)) e $x_{f}$ (Figura 8(b)). As medições foram feitas entre os sobressaltos das molas em relação às barras.

O paquímetro utilizado nas medições possui nônio de 20 divisões, portanto uma resolução de $0,05 \mathrm{~mm}$ e incerteza "instrumental" de medida igual a $0,025 \mathrm{~mm}$.

O resultado dessas medições estão reunidas na Tabela 5 e Tabela 6 para a viga 1 e viga 2 , respectivamente.

Conforme observado, os valores obtidos pela medição direta são muito próximos dos resultados obtidos pelo método dos nós. Considerando a incerteza da força elástica, podemos afirmar que o método aplicado neste trabalho é compatível com o modelo teórico do método dos nós. A Tabela 7 reúne os resultados para o comparativo para cada barra $b$ entre a força sobre a barra calculada pelo método dos nós $\left(F_{M N . b}\right)$ e a força sobre a barra obtida por medição direta via lei de Hooke $\left(F_{M D . b}\right)$. O erro relativo percentual foi calculado pela expressão

$$
\frac{F_{M D . b}-F_{M N . b}}{F_{M N . b}} \cdot 100 .
$$

Para facilitar a visualização da compatibilidade do método aplicado neste trabalho com o modelo teórico do método dos nós, a Figura 9 e Figura 10 apresentam os gráficos comparando as forças de ação sobre cada barra
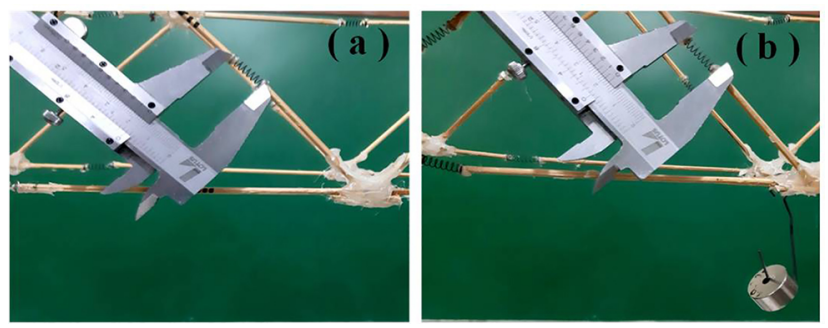

Figura 8: Medições do comprimento da mola (a) antes e (b) após a introdução da carga adicional $\boldsymbol{P}$.

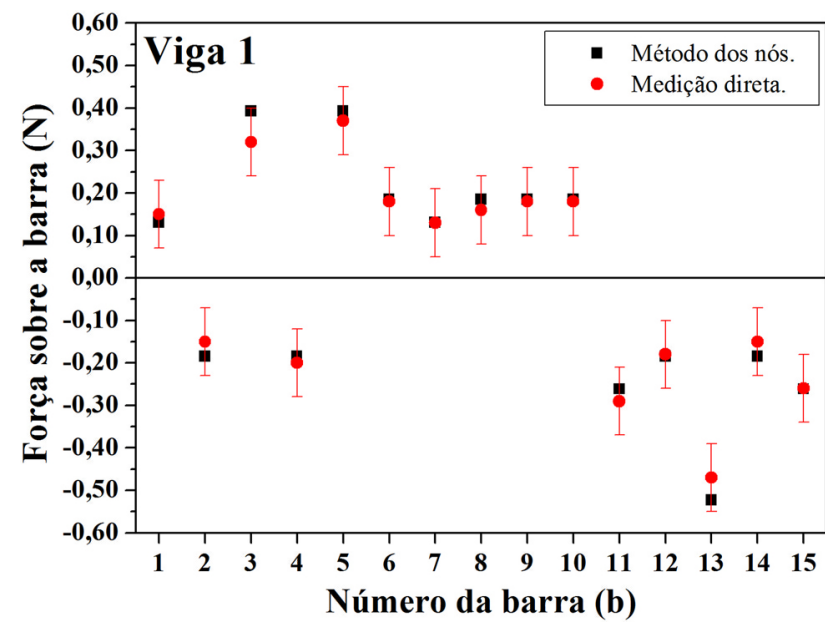

Figura 9: Força de ação sobre cada barra $b$ para viga 1. Os quadrados pretos representam os resultados pelo método dos nós e em círculos vermelhos por medição direta com as respectivas incertezas.

por medição direta e pelo método dos nós com suas respectivas incertezas para viga 1 e viga 2 .

Nota-se que as molas se comportam como "sensores" fornecendo resultados confiáveis, compatíveis com o modelo adotado pelos engenheiros em seus cálculos. Estes "sensores" permitem a visualização das forças axiais de compressão e tração atuantes na estrutura em tempo real. É importante ressaltar que a maquete didática experimental só é eficiente se as molas forem acopladas a todas as barras diagonais e longitudinais.

\section{Conclusão}

Na tentativa de contribuir para o processo de ensino e aprendizagem trouxemos uma ponte de varetas de bambu com molas acopladas na estrutura como proposta experimental para facilitar a compreensão das leis físicas que governam uma estrutura em treliças. 
Tabela 5: Resultados das forças por medição direta da viga 1. A numeração das barras seguem à apresentada na Figura 4, $k=$ $325,8(1) \mathrm{N} / \mathrm{m}$, a incerteza para as forças nas barras é $\delta F_{b}=0,08 \mathrm{~N}$ e a incerteza para deformação na mola é $\delta \Delta x=0,00025 \mathrm{~m}$.

\begin{tabular}{|c|c|c|c|c|c|c|}
\hline \multicolumn{7}{|c|}{ Viga 1} \\
\hline & $\mathrm{N}^{\circ}$ da Barra $(b)$ & $x_{\mathrm{i}}(\mathrm{m})$ & $x_{\mathrm{f}}(\mathrm{m})$ & $\Delta x_{\mathrm{b}}(\mathrm{m})$ & $F_{\mathrm{b}}=k \Delta x_{\mathrm{b}}(\mathrm{N})$ & Comportamento \\
\hline \multirow[t]{3}{*}{ Banzo superior } & 11 & 0,01830 & 0,01740 & $-0,00090$ & $-0,29$ & Compressão \\
\hline & 13 & 0,01475 & 0,01330 & $-0,00145$ & $-0,47$ & Compressão \\
\hline & 15 & 0,01560 & 0,01480 & $-0,00080$ & $-0,26$ & Compressão \\
\hline \multirow[t]{8}{*}{ Barras diagonais } & 2 & 0,01500 & 0,01455 & $-0,00045$ & $-0,15$ & Compressão \\
\hline & 9 & 0,01630 & 0,01685 & 0,00055 & 0,18 & Tração \\
\hline & 4 & 0,01660 & 0,01600 & $-0,00060$ & $-0,20$ & Compressão \\
\hline & 10 & 0,01630 & 0,01685 & 0,00055 & 0,18 & Tração \\
\hline & 6 & 0,01620 & 0,01675 & 0,00055 & 0,18 & Tração \\
\hline & 12 & 0,01455 & 0,01400 & $-0,00055$ & $-0,18$ & Compressão \\
\hline & 8 & 0,01600 & 0,01650 & 0,00050 & 0,16 & Tração \\
\hline & 14 & 0,01725 & 0,01680 & $-0,00045$ & $-0,15$ & Compressão \\
\hline \multirow[t]{4}{*}{ Banzo inferior } & 1 & 0,01690 & 0,01735 & 0,00045 & 0,15 & Tração \\
\hline & 3 & 0,01935 & 0,02035 & 0,00100 & 0,32 & Tração \\
\hline & 5 & 0,01860 & 0,01975 & 0,00115 & 0,37 & Tração \\
\hline & 7 & 0,01730 & 0,01770 & 0,00040 & 0,13 & Tração \\
\hline
\end{tabular}

Tabela 6: Resultados das forças por medição direta da viga 2 . A numeração das barras seguem à apresentada na Figura $4, k=$ $325,8(1) \mathrm{N} / \mathrm{m}$, a incerteza para as forças nas barras é $\delta F_{b}=0,08 \mathrm{~N}$ e a incerteza para deformação na mola é $\delta \Delta x=0,00025 \mathrm{~m}$.

\begin{tabular}{|c|c|c|c|c|c|c|}
\hline \multicolumn{7}{|c|}{ Viga 2} \\
\hline & \multicolumn{6}{|l|}{ Barra (b) } \\
\hline Barras longitudinais & 11 & 0,01600 & 0,01510 & $-0,00090$ & $-0,29$ & Compressão \\
\hline superiores (Banzos & 13 & 0,01510 & 0,01365 & $-0,00145$ & $-0,47$ & Compressão \\
\hline superiores) & 15 & 0,01610 & 0,01520 & $-0,00090$ & $-0,29$ & Compressão \\
\hline \multirow[t]{8}{*}{ Barras diagonais } & 2 & 0,01600 & 0,01545 & $-0,00055$ & $-0,18$ & Compressão \\
\hline & 9 & 0,01640 & 0,01695 & 0,00055 & 0,18 & Tração \\
\hline & 4 & 0,01550 & 0,01500 & $-0,00050$ & $-0,16$ & Compressão \\
\hline & 10 & 0,01610 & 0,01665 & 0,00055 & 0,18 & Tração \\
\hline & 6 & 0,01620 & 0,01670 & 0,00050 & 0,18 & Tração \\
\hline & 12 & 0,01710 & 0,01660 & $-0,00050$ & $-0,16$ & Compressão \\
\hline & 8 & 0,01640 & 0,01690 & 0,00050 & 0,16 & Tração \\
\hline & 14 & 0,02045 & 0,01990 & $-0,00055$ & $-0,18$ & Compressão \\
\hline \multirow{4}{*}{$\begin{array}{l}\text { Barras longitudinais } \\
\text { inferiores (Banzos } \\
\text { inferiores) }\end{array}$} & 1 & 0,01630 & 0,01670 & 0,00040 & 0,13 & Tração \\
\hline & 3 & 0,01610 & 0,01715 & 0,00105 & 0,34 & Tração \\
\hline & 5 & 0,01810 & 0,01905 & 0,00095 & 0,31 & Tração \\
\hline & 7 & 0,01765 & 0,01810 & 0,00045 & 0,15 & Tração \\
\hline
\end{tabular}

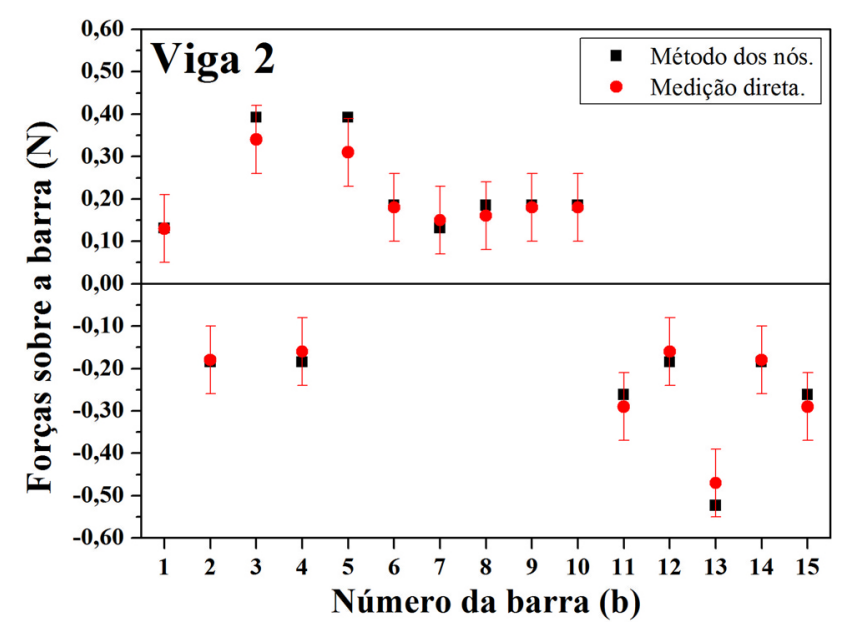

Figura 10: Força de ação sobre cada barra b para viga 2. Os quadrados pretos representam os resultados pelo método dos nós e os círculos vermelhos por medição direta com as respectivas incertezas. 


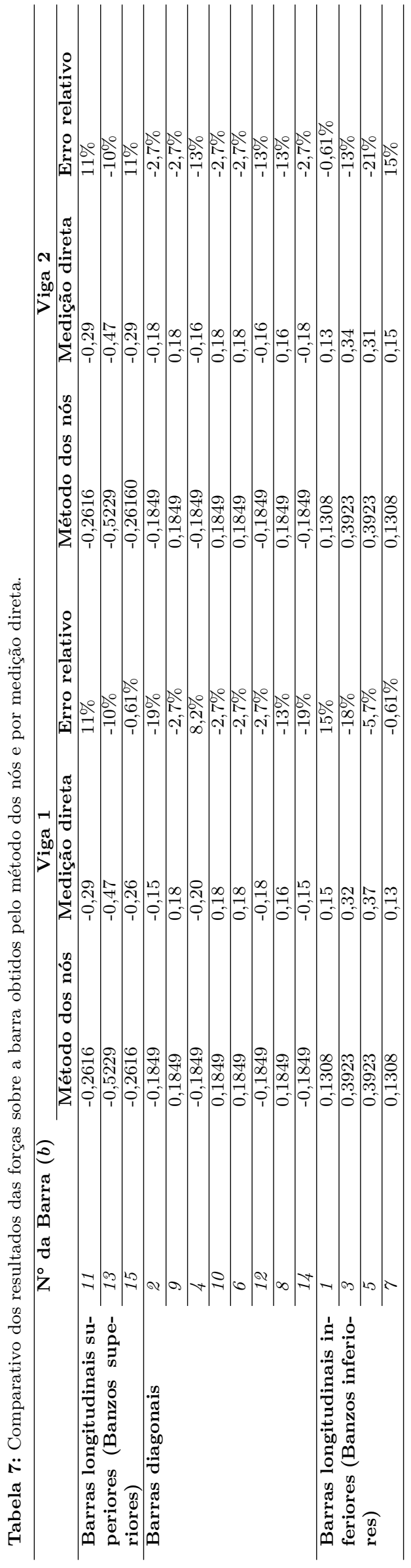

Comparamos o modelo pelo método dos nós e pela medição direta. Note na Tab. 7 que na barra longitudinal (banzo) inferior 5, da viga 2 , foi encontrado um erro relativo percentual de $-21 \%$. Se tomarmos o cálculo pelo método dos nós da força exercida sobre essa barra, $F_{M N .5}=0,3923 \mathrm{~N}$ e dividirmos pelo valor de $k=325,8$ $\mathrm{N} / \mathrm{m}$, encontramos $F_{M N .5} / k=0,001204 \mathrm{~m}$. Da Tab. 6, para essa mesma barra, $\Delta x_{5}=0,00095 \mathrm{~m}$. Fazendo a diferença $F_{M N .5} / k-\Delta x_{5}=0,00025 \mathrm{~m}$, ou seja, a mesma incerteza "instrumental" do paquímetro. De qualquer forma, pequenas torções nas molas foram observadas em algumas barras da treliça. Na barra longitudinal (banzo) inferior 3 , da viga 1 , à esquerda do centro da Figura 8(b), podemos visualizar uma das torções ocorrida em algumas molas. Para essa barra, na Tab. 7 registramos um erro relativo percentual de $-18 \%$, porém note que $F_{M N .3} / k=0,001204 \mathrm{~m}$ e pela Tabela $5, \Delta x_{3}=0,00100$ $\mathrm{m}$. Ao fazer a diferença $F_{M N_{3}} / k-\Delta x_{3}=0.00020 \mathrm{~m}$, ou seja, um valor menor que a incerteza "instrumental" do paquímetro. Concluímos que os resultados encontrados pelo método dos nós e por medição direta utilizando a lei de Hooke são semelhantes, podendo assim, a maquete didática apresentada servir de atividade experimental confiável para fins didáticos.

Conforme enunciamos, nossa estrutura com molas é uma maquete didática experimental para visualização e medida da atuação das forças nas barras. Outro fator a considerar é que qualquer questionamento ou hipótese levantado pelos estudantes acerca do comportamento da estrutura, como por exemplo, cargas deslocadas do eixo, cargas no banzo superior e etc. poderão ser facilmente calculadas modificando a posição de $P$ na estrutura e se fazer novas medidas. Esse tipo de maquete poderá ser montada pelos alunos com barras rígidas para experimentarem suas respectivas estruturas a ensaios de resistência à ruptura.

Como sugestão, para uma intervenção didática utilizando a experimentação proposta nesse artigo, é importante destacar concepções epistemológicas que sustentam a experimentação e práticas pedagógicas com orientação CTS. Por exemplo, relacionar a Ciência, Engenharia e Sociedade, por meio de discussões atreladas às questões de impactos ambientais, impactos estéticos e impactos econômicos das pontes em zonas urbanas.

\section{Material suplementar}

O seguinte material suplementar está disponível online:

Apêndice A - Cálculo das forças nas barras pelo método dos nós.

\section{Referências}

[1] M.O.A. da Silva e C.D.P. Portela, Revista Científica Interdisciplinar 3, 5 (2018).

[2] M.P. Belançon, Revista Brasileira de Ensino de Física 39, e4001 (2017). 
[3] M.S. Taha, C.S.C. Lopes e E.L. Soares, V. Folmer, Experiências em Ensino de Ciências 11, 138 (2016).

[4] V.M. de Souza, S.S. Rodrigues e M.G. Ramos, Indagatio Didactica 8, 584 (2016).

[5] N.T. Massoni, Caderno Brasileiro de Ensino de Física 31, 258 (2014).

[6] D.B. Bigoni, F. Dal Corso, D. Misseroni e M. Tommasini, Eur. J. Phys. 33, 1179 (2012).

[7] F.P. Beer, E.R. Johnston e D.F. Mazurek. Mecânica Vetorial para Engenheiros: Estática. (Ed. McGraw-Hill, Porto Alegre, 2019), 11ª ed. 